\title{
Gamma-ray burst phenomenology explained through the blast wave model
}

\author{
C.D. Dermer \\ Code 7653, Naval Research Laboratory, Washington, DC 20375-5352, U.S.A. \\ e-mail: dermer@burst.nrl.navy.mil
}

Received December 29, 1998; accepted March 14, 1999

\begin{abstract}
The essential physics of the blast wave model is contained in a formula recently proposed to describe the temporal evolution of the nonthermal synchrotron spectra from gamma-ray bursts (GRBs). This formula accounts for many of the well-established empirical trends in GRB physics, including the basic form of the fast-rise, gentledecay FRED-type GRB light curves, the characteristic hard-to-soft spectral evolution and intensity-hardness correlations of GRB light curves, and the recently uncovered relations between the $\nu F_{\nu}$ peak energies and the photon and energy fluences. On the basis of this formula, we predict the tendency for pulses to align at high energies and to lag at low energies. The overall GRB duration distribution is understood when triggering properties of GRB detectors are considered for fireballs with a range of baryon loading parameters. The existence of new classes of clean and dirty fireballs are implied. Short timescale variability of GRB light curves is attributed to density variations in the circumburster medium (CBM).
\end{abstract}

Key words: gamma-rays: bursts

\section{Introduction}

In the fireball/blast wave model, the impulsive release of energy by a cosmic source forms an expanding fireball whose energy is transformed into a relativistic blast wave, which attains an initial Lorentz factor $\Gamma_{0}>>1$ determined by the baryon loading of the fireball (Blandford \& McKee 1976; Rees \& Mészáros 1992). The power-law behaviors of the X-ray and optical afterglows discovered as a result of the Beppo-SAX mission are simply explained as the Doppler-shifted nonthermal lepton synchrotron emissions (e.g., Katz 1994; Tavani 1997) from a decelerating blast wave (e.g., Waxman 1997; Vietri 1997).

Send offprint requests to: C. Dermer
Less attention has been devoted to using this model to understand the large body of hard X-ray/soft $\gamma$-ray phenomenology of GRBs. Here we summarize our recent work which shows how the phenomenology of GRBs can be understood in the context of a simple formula based on the blast wave model.

\section{Generalization of the Band function}

The "Band" function (Band et al. 1993) is a fourparameter phenomenological fit consisting of two smoothly connected power laws that is widely used to fit GRB spectra. Typically, the rising $\nu F_{\nu}$ indices $v$ below the peak $\nu F_{\nu}$ energy $\epsilon_{\text {peak }}$ have measured values $v \cong 1$, and the falling indices $\delta$ have values $\delta \approx 0-0.5$, where $v, \delta>0$ by definition. The prediction of the nonthermal synchrotron shock model is that $v \leq 4 / 3$ as a result of the intrinsic synchrotron emissivity spectrum.

We have proposed (Dermer et al. 1999a) a temporallyevolving generalization of the Band form based on the physics of the blast wave model. This formula is essentially a Band function with both the amplitude and $\epsilon_{\text {peak }}$ changing with time. Nine parameters in all are necessary to define a model, namely the (i) total energy $E_{0}$ of the explosion; (ii) $\Gamma_{0}$; (iii) the equipartition factor $q$; (iv) the density $n_{0}$ and (v) radial-gradient index $\eta$ of the CBM (see below); (vi) the redshift $z$ of the source; (vii) the radiative regime $g$ describing the blast wave deceleration $\Gamma \propto x^{-g}$; and the spectral indices (viii) $v$ and (ix) $\delta$. We also assume that $q, v$, and $\delta$ are independent of time. This formula lacks self-consistency in the sense that a magnetic field of sufficient strength must be present for the blast wave to be found in a particular radiative regime. These limitations of the model can only be addressed through detailed numerical simulations (e.g., Chiang \& Dermer 1999).

\section{GRB phenomenology explained}

Some $20-30 \%$ of GRBs show the FRED (Fast Rise/ Exponential Decay) behavior. For a simple FRED-type 
GRB, or for well-defined peaks within a burst light curve, hard-to-soft evolution is usually measured (e.g., Norris et al. 1986). A hardness-intensity correlation is also generally observed for well-defined pulses within a GRB (Golenetskii et al. 1983). Both of these viewpoints are probably correct insofar as each well-defined pulse in a GRB time profile is accompanied by a spectral hardening and subsequent softening (Ford et al. 1995). When $\epsilon_{\text {peak }}$ is plotted against photon or energy fluence, then $\epsilon_{\text {peak }}$ decays as a power-law or a broken power law (Liang \& Kargatis 1996; Crider et al. 1998). We (Dermer et al. 1999b) have recently shown how this behavior can be understood in terms of the analytic function described above.

Our model spectrum makes definite predictions if one assumes that the FRED-type profile of GRB peaks is a consequence of a smooth CBM that is reasonably well-described by a density $n(x) \propto n_{0} x^{-\eta}$. At early times $t \ll t_{\mathrm{d}}$, where the deceleration time scale $t_{\mathrm{d}} \propto \Gamma_{0}^{-8 / 3}\left(E_{0} / n_{0}\right)^{1 / 3}$, the temporal profile of the rising flux $\left(\phi \propto t^{-\chi}\right)$ of a decelerating blast wave exhibits temporal indices $|\chi|=2-\eta+\eta v / 2$ at low energies $\epsilon \ll \mathcal{E}_{0}$ and $|\chi|=2-\eta-\eta \delta / 2$ at high energies $\epsilon \gg \mathcal{E}_{0}$. Here $\mathcal{E}_{0} \equiv \epsilon_{\text {peak }}\left(t_{\mathrm{d}}\right)$. Because $\mathcal{E}_{0}, v$, and $\delta$ can all be measured, fast timing over a broad energy band should give shifts in the temporal indices of the rising portion of the GRB time profiles approaching these limits. We also predict (Dermer et al. 1999b) that the time of the peak flux of a well-defined GRB pulse measured at different photon energies $\left(\varepsilon<\mathcal{E}_{0}\right)$ should follow the relation

$t_{\mathrm{p}}(\epsilon)=\frac{t_{\mathrm{d}}}{2 g+1}\left[2 g+\left(\frac{\epsilon}{\mathcal{E}_{0}}\right)^{-(2 g+1) /(4 g+\eta / 2)}\right]$.

Thus the peaks should align at high energies and lag at lower energies. The peak alignment in GRBs is quite well-documented at $\gtrsim 50 \mathrm{keV}$ energies, but the character of peak alignment and lagging is less well-studied at $\lesssim 20 \mathrm{keV}$ (see, however, Piro et al. 1998; Connors \& Hueter 1998).

Perhaps the most crucial test for a model of GRBs is to explain the overall GRB duration distribution, including the strong evidence for bimodality (Kouveliotou et al. 1996). In our recent work (Böttcher \& Dermer 1999) we show that the GRB duration distribution measured with BATSE and other instruments is a consequence of the emission properties of blast waves, the triggering criteria of GRB detectors, and the range of values of $\Gamma_{0}$ and $E_{0}$.

\section{Prediction of new fireball types}

In Dermer et al. (1999a), we found that the behavior of the temporally-evolving emission is robust to orders-ofmagnitude changes in all parameter values except for $q$ and $\Gamma_{0}$. The effect of changing $q$ is to translate $P(\epsilon, t)$ horizontally along the $\epsilon$ axis by the factor $q$. The value of $q$ can be constrained, at least during the prompt phase of the GRB, by spectral modeling.
The observability of a fireball is most profoundly affected by the value of $\Gamma_{0}$. For clean fireballs with small baryon loading $\left(\Gamma_{0} \gg 300\right)$, we find that GRBs are intense, subsecond, medium-to-high energy ( $\gg 10 \mathrm{MeV}$ ) $\gamma$-ray events, and are difficult to detect because of inadequate photon counts given the insufficiently large effective areas $\left(\sim 10^{3} \mathrm{~cm}^{2}\right)$ of $>100 \mathrm{MeV} \gamma$-ray detectors such as EGRET on CGRO and deadtime limitations. Dirty fireballs $\left(\Gamma_{0} \ll 300\right)$ produce transient emissions which are longer lasting and most luminous at X-ray energies and below, but these events are lost behind the glow of the $\mathrm{X}$-ray and lower-energy background radiations except for rare serendipitous detections by pointed instruments.

\section{Short timescale variability of prompt and delayed GRB emissions}

One of the central problems in GRB studies is to explain the diversity of GRB time profiles. We argued above that the simple FRED-type profiles can be understood by blast wave deceleration in a uniform or smoothly varying CBM where the density $n(x) \propto x^{-\eta}$. To explain the temporal variability of GRBs, we (Dermer \& Mitman 1999) recently considered a highly-structured cloudy medium on a large range of distance scales. Column densities of only $10^{16}-10^{20} \mathrm{~cm}^{-2}$ are needed to efficiently extract the energy from a relativistic blast wave, so the properties of such a CBM medium are not extreme. A CBM that is inhomogeneous on small scales also breaks the symmetry on angular scales $\ll 1 / \Gamma_{0}$, so that the objections of Fenimore and colleagues are met regarding emission from a single relativistic shell (Fenimore et al. 1996).

\section{References}

Band D., et al., 1993, ApJ 413, 281

Blandford R.D., McKee C.F., 1976, Phys. Fluids 19, 1130

Böttcher M., Dermer C.D., 1999, ApJ (submitted)

Chiang J., Dermer C.D., 1999, ApJ 512, 699

Connors A., Heuter G.J., 1998, ApJ

Crider A., Liang E.P., Preece R.D., 1998, in Gamma Ray Bursts: 4th Huntsville Sympsium, Meegan C.A. et al. (eds.). New York: AIP, p. 63

Dermer C.D., Chiang J., Böttcher M., 1999a, ApJ 513, 656

Dermer C.D., Böttcher M., Chiang J., 1999b, ApJL 515, L49

Dermer C.D., Mitman K., 1999, ApJ 513, L5

Fenimore E.E., Madras C., Nayakshin S., 1996, ApJ 473, 998

Ford L.A., et al., 1995, ApJ 439, 307

Golenetskii S.V., et al., 1983, Nat 306, 451

Katz J.I., 1994, ApJ 432, L107

Kouveliotou C., et al., 1993, ApJ 413, L101

Liang E.P., Kargatis V., 1996, Nat 381, 49

Norris J., et al., 1986, ApJ 301, 213

Piro L., et al., 1998, A\&A 329, 906

Rees M.J., Mészáros P., 1992, MNRAS 258, 41P

Tavani M., 1997, ApJ 483, L87

Vietri M., 1997, ApJ 478, L9

Waxman E., 1997, ApJ 485, L5 